\title{
Velocity analysis in homogeneous VTI media using SVD-semblance
}

\author{
Rubenvaldo M. Pereira, João Carlos R. Cruz and João dos S. Protázio, UFPA, Brazil
}

Copyright 2016, SBGf - Sociedade Brasileira de Geofísica.

This paper was prepared for presentation at the VII Brazilian Symposium on geophysics, held in Ouro Preto, Brazil, October 25-27, 2016. Contents of this paper were reviewed by the Technical Committee of the VII SimBGf and do not necessarily represent any position of the SBGf, its officers or members. Electronic reproduction or storage of any part of this paper for commercial purposes without the written consent of The Brazilian Geophysical Society is prohibited.

\begin{abstract}
Velocity analysis in prestack reflection data on CMP gathers is traditionally perform using semblance coherence measure with hyperbolic moveout curves. However, due to the assumptions made in its development, this measure is inaccurate in estimating parameters in sections with long-spread, anisotropy, and in the presence of amplitude variation with offset (AVO), especially in the presence of polarity reversals. Nonhyperbolic moveout approximations are commonly used to approximate moveout on surveys with long offsets in anisotropic media. Among these, the shifted hyperbola approximation is considered quite valid to model traveltime reflections in CMP gather data with large offsets obtained in VTI media. Amplitude variations with offset, in the presence of reverse polarity may be introduced in velocity analysis, modifying the traditional semblance algorithm. Singular value decomposition (SVD) applied to the semblance reduces the influence of AVO even in the presence of noise. In this paper we perform velocity analysis based on semblance SVD with shifted hyperbole approximation in estimating parameters in anisotropic media VTI (transversely isotropic with a vertical symmetry axis) in data free and with noise. The results suggest the validity, accuracy and robustness, technique to estimate parameters in such media.
\end{abstract}

\section{Introduction}

Velocity analysis in seismic sections is performed using Coherence measures in general semblance. However, in seismic data with high amplitude variation along the seismic events and offset-depth ratio greater than one, the semblance loses precision in estimation of parameters. This has led to consider changes to the semblance in order to suit it to seismic sections with anisotropy, long offsets, variations in amplitude with the offset and reverse polarization.

Several nonhyperbolic moveout curves in CMP sections with long offsets have been proposed to approximate traveltime in VTI media (Tsvankin and Thomsen (1994); Alkhalifah and Tsvankin (1995); Fomel (2004)). Methods for performing semblance-based velocity analysis using nonhyperbolic approximations, have been presented by Alkhalifah (1997)and Grechka and Tsvankin (1998). Using approximations by rational interpolation Douma and Calvert (2006) and Douma and Baan (2008) presented an accurate method for performing semblance-based velocity analysis in homogeneous VTI layered media.
Sarkar et al. (2001) and Sarkar et al. (2002) presented $A B$ and $A K$-semblance algorithms in order to make the semblance, sensitive to variations in phase and amplitude with offset. Their experiments showed that the new measures were robust to deal with AVO anomalies and reverse polarity. Yan and Tsvankin (2008) extended the AK-semblance algorithm for VTI media and large offsets using nonhyperbolic moveout equation of Alkhalifah and Tsvankin (1995). Using singular value decomposition of the matrix formed by the windowed data in semblance, Ursin et al. (2014) showed an adaptation to semblance, precise and robust enough to handle data with large amplitude variations with the offset.

In this paper, we present the velocity analysis based on SVD-semblance algorithm with nonhyperbolic moveout aproximation shifted hyperbola in order to measure the accuracy and robustness in the parameter estimation in homogeneous VTI media.

\section{Wave propagation in VTI media}

TI media are characterized by five elastic parameters, density-normalized: $a_{11}, a_{13}, a_{33}, a_{44}$ and $a_{66}$. The P-wave propagation in VTI media has phase velocity equation, as a function of the phase angle $\theta$ given by Gassmann (1964); Thomsen (1986):

$$
\begin{aligned}
\frac{v^{2}(\theta)}{v_{P z}^{2}} & =1+\varepsilon \sin ^{2} \theta \\
& +\sqrt{1+\frac{4 \sin ^{2} \theta}{f}\left(2 \delta \cos ^{2} \theta-\varepsilon \cos 2 \theta\right)+\frac{4 \varepsilon^{2} \sin ^{4} \theta}{f^{2}}},
\end{aligned}
$$

where $\varepsilon$ and $\delta$ are dimensionless parameters Thomsen (1986) and $f=1-\frac{v_{S z}^{2}}{v_{P z}^{2}}$, with $v_{P z}=\sqrt{a_{33}}$ and $v_{S} z=\sqrt{a_{44}}$ the $\mathrm{P}$ - and S-wave vertical velocity, respectively.

In anisotropic media the group velocity equation and the group angle equation, are difficult to explain. However, its square magnitude, $V$, and the group angle $\Theta$, can be obtained from the phase velocity, $v$, and phase angle, $\theta$, respectively, as Berryman (1979):

$$
V^{2}(\Theta)=v^{2}(\theta)+\left[\frac{d v(\theta)}{d \theta}\right]^{2}
$$

and

$$
\tan (\Theta)=\frac{\tan (\theta)+\frac{1}{v(\theta)} \frac{d v(\theta)}{d \theta}}{1-\frac{\tan (\theta)}{v(\theta)} \frac{d v(\theta)}{d \theta}},
$$

Considering a flat reflective interface to a depth $z$, the wave propagation traveltime in CMP gather as a function of the offset $x$ in homogeneous VTI media, is nonhyperbolic and it is given by:

$$
t^{2}(x)=\frac{4 z^{2}+x^{2}}{V^{2}(\Theta)},
$$

However, due to their representation depends explicitly of the group velocity, moveout approximations are often 
used on the steps of seismic processing. Moveout approximations based on Taylor series expansion are widely used in seismic processing. The shifted hyperbola approximation Malovichko (1978) is an accurate moveout curve of the fourth order Castle (1994), ie it is nonhyperbolic. Fomel (2004) explained, analytically, the shifted hyperbola approximation for homogeneous VTI media as:

$$
t_{P}^{2}(x) \approx \frac{3+4 \eta}{4(1+\eta)} t_{P h}^{2}(x)+\frac{1}{4(1+\eta)} \sqrt{t_{P h}^{4}(x)+\frac{16 \eta(1+\eta)}{(1+2 \eta)} \frac{t_{P z}^{2} x^{2}}{v_{P n}^{2}}} .
$$

where $t_{P h}^{2}(x)=t_{P z}^{2}+\frac{x^{2}}{(1+2 \eta) v_{P n}^{2}}$, with: $t_{P z}$ the P-wave twoway vertical traveltime, $v_{P n}=v_{P n}(1+2 \delta)$ is the normal moveout velocity, and $\eta=\frac{\varepsilon-\delta}{1+2 \delta}$ the anelliptic parameter Alkhalifah and Tsvankin (1995).

\section{Velocity analysis in VTI media}

Seismic data in a CMP gather with $N$ traces, basedsemblance velocity analysis is performed to estimate $t_{z}$ and $v_{n}$, maximizing the functional Taner and Koehler (1969):

$$
S\left(t_{z}, v\right)=\frac{\sum_{\tau=t_{z}-T / 2}^{t_{z}+T / 2}\left[\sum_{x} \mathbf{D}(\tau, x)\right]^{2}}{N \sum_{\tau=t_{z}-T / 2}^{t_{z}+T / 2} \sum_{x} \mathbf{D}^{2}(\tau, x)}
$$

where $\tau$ is the two-way vertical traveltime in a time window, $T$, centered at time $t_{z}$ and $\mathbf{D}(t, x)$ is a data window sampled by a moveout curve $t(\tau, v)$ with $v$ being the stacking velocity. Generally semblance uses hyperbolic moveout curve and is accurate in estimating parameters for data without AVO and offset-depth ratio $x / z$ values at most one.

However, current seismic processing techniques have used as a model of subsurface anisotropic media, especially anisotropic VTI media. This has required adjustments to processing steps in such a way that they can incorporate the wave propagation characteristics in such lithologic models. VTI media are widely used as a model of the subsurface seismic exploration because they describe more accurately the wave propagation in hydrocarbon reserves,for exemple, in shales and periodic thin layering (thin isotropic layers).

\section{Semblance SVD homogeneus VTI media}

Consider now the $N_{t} \times N_{x}$ data matrix $\mathbf{D}(\tau, x)$ :

$$
\mathbf{D}=\mathbf{W}+\mathbf{N},
$$

where $\mathbf{W}$ is the $N_{t} \times N_{x}$ signal matriz and $\mathbf{N}$ is the $N_{t} \times N_{x}$ noise matriz. In this way, the semblance 6 is rewrite as:

$$
S=\frac{\|\mathbf{W}\|_{F}^{2}}{\|\mathbf{D}\|_{F}^{2}},
$$

where $\|\mathbf{D}\|_{F}$ is the Frobenius norm, defined as:

$$
\|\mathbf{D}\|_{F}=\sum_{i=1}^{N} \sum_{j=1}^{N}\|\mathbf{D}\|^{2}
$$

and $\mathbf{W}$ is the signal matrix:

$$
\mathbf{W}=\mathbf{s e}^{T},
$$

which is the product between the signal vetor $\mathbf{s}$ and the transposed of $N_{x} \times 1$ scattered vector: $\mathbf{e}^{T}=[1,1, \ldots, 1]$.
Calculating the SVD (singular value decomposition) of the data matrix Golub and Van Loan (1996):

$$
\mathbf{D}=\mathbf{U} \Sigma \mathbf{V}^{T}=\sum_{i=1}^{N_{t}} \mathbf{u}_{k} \sigma_{k} \mathbf{v}_{k}^{T},
$$

where $\mathbf{u}_{k}$ and $\mathbf{v}_{k}$ are the $N_{t} \times 1$ and $N_{x} \times 1$ ortonormal vectors, respectivaly, and $\sigma$ its singular values chosen such that: $\sigma_{1} \geq \sigma_{2} \geq, \ldots, \geq \sigma_{N_{t}}$, to $N_{t} \leq N_{x}$. The semblance can then be rewritten asUrsin et al. (2014):

$$
S=\frac{\sum_{k=1}^{N_{t}} \sigma_{k}^{2}\left\|\mathbf{D} \mathbf{v}_{k}\right\|^{2}}{N_{x} \sum_{k=1}^{N_{t}} \sigma_{k}^{2}}
$$

However, assuming that the shape signal remains the same in all traces, but with the amplitude varying with the offset, the signal matrix can take the form:

$$
\mathbf{W}=\mathbf{s} \mathbf{a}^{T}
$$

where $\mathbf{a}^{T}=\left[a_{1}, a_{2}, \ldots, a_{N x}\right]$, and $\mathbf{a}$ a vector with amplitudes in each channel. Golub and Van Loan (1996) showed that a least squares estimation of an array, is a first selfimage. Thus, the signal matrix can be decomposed as $\mathbf{W}=$ $\mathbf{u}_{1} \sigma_{1} \mathbf{v}_{1}^{t}$, and semblance operator modified as Gersztenkorn and Marfurt (1999); Ursin et al. (2014):

$$
S_{S V D}=\frac{\sigma_{1}^{2}}{\sum_{k=1}^{N_{t}} \sigma_{k}^{2}}
$$

hereinafter called SVD semblance.

\section{Velocity Analisy using SVD semblance}

In this experiment are performed velocity analysis based on semblance SVD, with the shifted hyperbola nonhyperbolic approximation (5) considering as a model of subsurface the VTI shale Greenhorn Jones and Wang (1981), whose elastic parameters normalized by the density are: $a_{11}=14.47 \mathrm{~km}^{2} / \mathrm{s}^{2}, a_{13}=4.51 \mathrm{~km}^{2} / \mathrm{s}^{2}, a_{33}=9.57 \mathrm{~km}^{2} / \mathrm{s}^{2}$ and $a_{55}=2.28 \mathrm{~km}^{2} / \mathrm{s}^{2}$. The seismic sections was obtained considering a reflective interface located at a depth $z=$ $1,0 \mathrm{~km}$, two way traveltime $t_{z}=0,6464 \mathrm{~s}$, velocities $v_{P n}=$ $2,9336 \mathrm{~km} / \mathrm{s}, v_{P x}=3,8045 \mathrm{~km} / \mathrm{s}$ and parameter $\eta=0,3409$. The Figure 1 present the synthetic sismograms where the traveltime was the sampled at $\Delta t=4 m s$ and the signature source is the Gabor wavelet with frequency $f=40 \mathrm{~Hz}$. In Figure 1 (a), (b) and (c) there are variation in the noise level.

First, the velocity analysis is performed on data for AVO variation ans free noise. The Figure 2 illustrate the $v_{P n}$ versus $v_{P x}$ SVD semblance maps and the results are shown in Table 1. What can be seen is that the velocity analysis is quite accurate in parameter estimates, because the relative error in estimating the velocity $v_{P n}$ does not exceed $0.4 \%$ and the relative error of the $\eta$ parameter is less than $2 \%$. It is noted also that the value of maximum SVD semblance is approximately 1.

Then, experiments are performed on data with AVO and variation in the values of $s / n$. The Figure ?? illustrate the $v_{P n}$ versus $v_{P x}$ SVD semblance obtained using shifted hyperbola nonhyperbolic approximation to $s / n=$ 15. The results are shown in Table ??. What can be observed, again is that the velocity analysis is quite accurate in parameter estimates, because the relative error in estimating the velocity $v_{P n}$ does not exceed $0.4 \%$, the 
Figure 1: CMP sintético (a) sem ruído, (b) com razão sinalruído igual a 15 e (c) com razão sinal-ruído igual a 5, obtido no folhelho VTI Greenhorn para $x / z=4,0$.

(a)

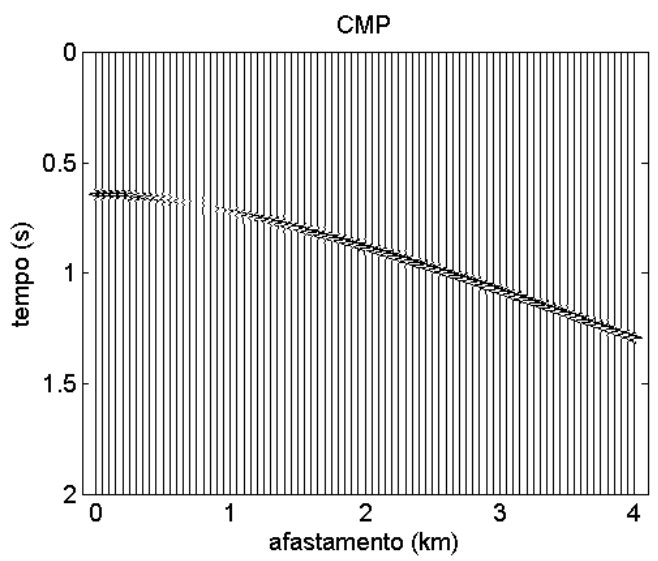

(b)

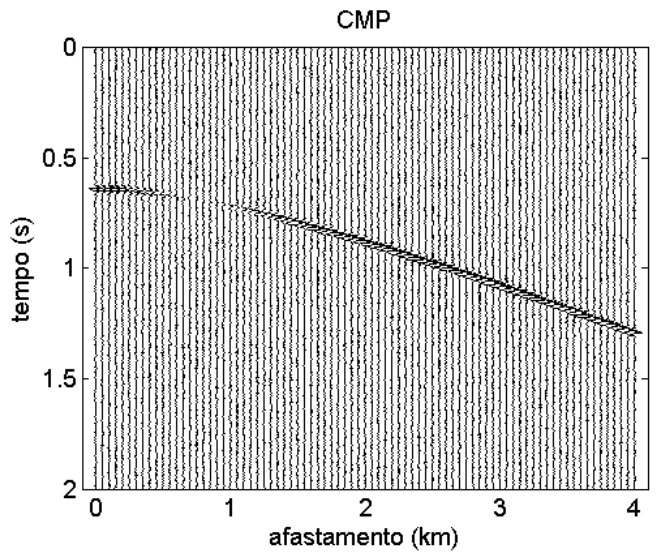

(c)

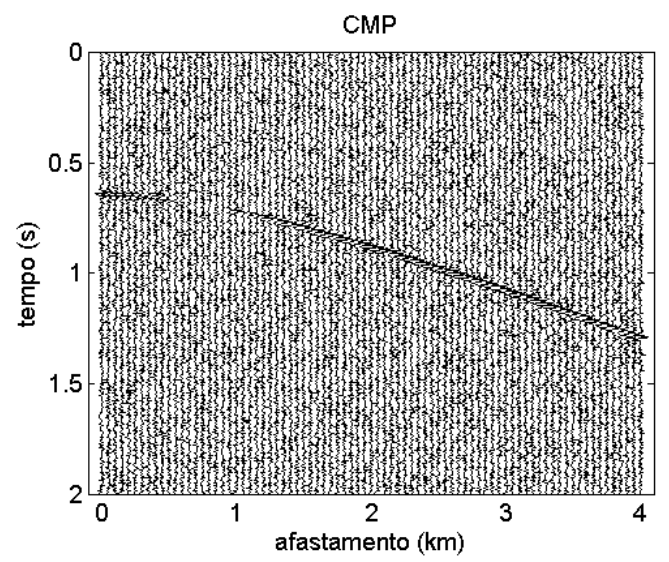

relative error of the $\eta$ parameter is less than $2 \%$ and the value of maximum SVD semblance is approximately 1 .

Finally, the Figure ?? illustrate the $v_{P n}$ versus $v_{P x}$ SVD semblance obtained using shifted hyperbola nonhyperbolic approximation to $s / n=5$. The results are shown in Table ??. What can be observed, again is that the
Figure 2: $v_{P n}$ versus $v_{P x}$ SVD emblance map, using Shifted hyperbola aproximation (5) to estimating velocities and $\eta$ parameter in datas with AVO and free noise, obtained in VTI Greenhorn shale, to $x / z=4,0$. Yellow cirle - exact value and white losango - approximate value.

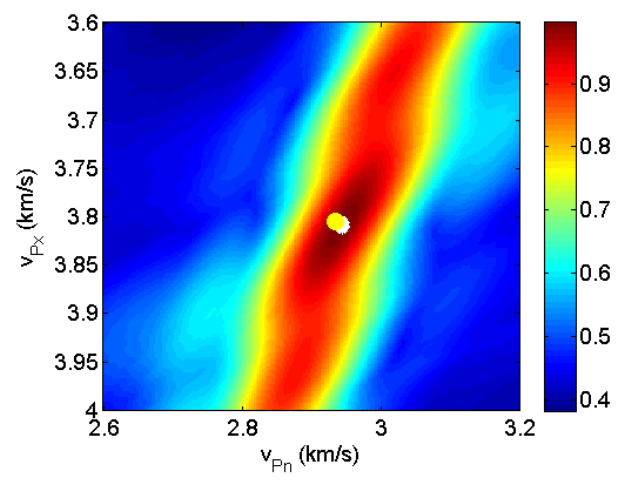

Table 1: relative error in estimated velocities $v_{P n}, v_{P x}$ and $\eta$ parameter, and maximum SVD semblance; obtained from velocity analysis in data with AVO and free noise, in the VTI Greenhorn shale to $x / z=4,0$.

\begin{tabular}{|c|c|c|c|}
\hline Error $v_{P n}(\%)$ & Error $v_{P x}(\%)$ & Error $\eta(\%)$ & $S_{\max }$ \\
\hline \hline 0.3449 & 0.1195 & 1.0964 & 0.9984 \\
\hline \hline
\end{tabular}

Figure 3: $v_{P n}$ versus $v_{P x}$ SVD emblance map, using Shifted hyperbola aproximation (5) to estimating velocities and $\eta$ parameter in datas with AVO and $s / n=15$, obtained in VTI Greenhorn shale, to $x / z=4,0$. Yellow cirle - exact value and white losango - approximate value.

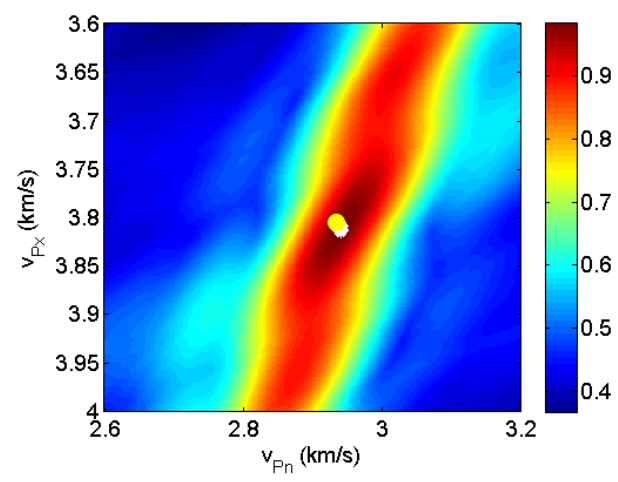

Table 2: relative error in estimated velocities $v_{P n}, v_{P x}$ and $\eta$ parameter, and maximum SVD semblance; obtained from velocity analysis in data with AVO and $s / n=15$, in the VTI Greenhorn shale to $x / z=4,0$.

\begin{tabular}{|c|c|c|c|}
\hline Error $v_{P n}(\%)$ & Error $v_{P x}(\%)$ & Error $\eta(\%)$ & $S_{\max }$ \\
\hline \hline 0.2421 & 0.1723 & 0.3328 & 0.9855 \\
\hline \hline
\end{tabular}

velocity analysis is quite accurate in parameter estimates, because the relative error in estimating the velocity $v_{P n}$ does not exceed $0.7 \%$, the relative error of the $\eta$ parameter is approximately $4 \%$ and the value of maximum SVD semblance is approximately 1 . 
Figure 4: $v_{P n}$ versus $v_{P x}$ SVD emblance map, using Shifted hyperbola aproximation (5) to estimating velocities and $\eta$ parameter in datas with AVO and $s / n=5$, obtained in VTI Greenhorn shale, to $x / z=4,0$. Yellow cirle - exact value and white losango - approximate value.

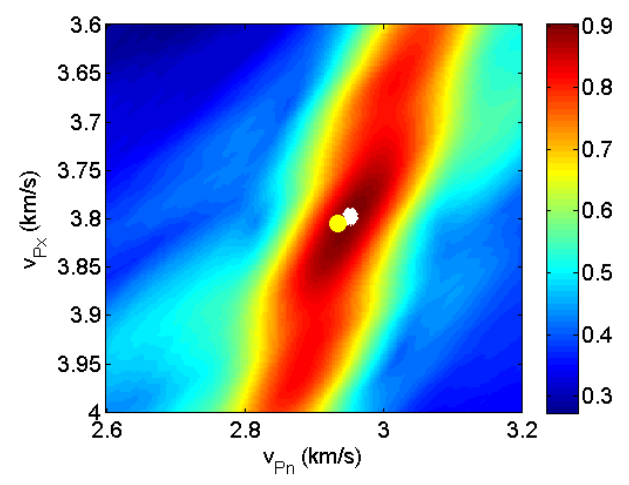

Table 3: relative error in estimated velocities $v_{P n}, v_{P x}$ and $\eta$ parameter, and maximum SVD semblance; obtained from velocity analysis in data with AVO and $s / n=5$, in the VTI Greenhorn shale to $x / z=4,0$.

\begin{tabular}{|c|c|c|c|}
\hline Error $v_{P n}(\%)$ & Error $v_{P x}(\%)$ & Error $\eta(\%)$ & $S_{\max }$ \\
\hline \hline 0.2421 & 0.1723 & 0.3328 & 0.9855 \\
\hline
\end{tabular}

\section{Results and Conclusions}

We have presented an adaptation of SVD semblance operator to estimate speed and parameter in VTI media. In general, the SVD semblance with Shifted hyperbola (5) nonhyperbolic approximation is a good option in velocity analysis to estimate velocyties: $v_{P n}$ and $v_{P x}$, and $\eta$ parameter in VTI data with AVO variation and polarity reversed. Experiments with and without noise and $x / z=$ 4,0 , showed that the SVD semblance is quite accurate and robust in parameter estimates. Thus, we conclude that the SVD semblance addition to mitigating the effects of AVO variation also acts as a noise filter.

\section{Acknowledgments}

The authors would like to thank the Project UFPa.

\section{References}

Alkhalifah, T., \& Tsvankin, I., 1995, Velocity analysis for transversely isotropic media: Geophysics, 60 , no. 5, 1550-1566.

Alkhalifah, T., 1997, Velocity analysis using nonhyperbolic moveout in transversely isotropic media: Geophysics, 62 , no. $6,1839-1854$.

Berryman, J. G., 1979, Long-wave elastic anisotropy in transversely isotropic media: Geophysics, 44, no. 5, 896-917.

Castle, R. J., 1994, A theory of normal moveout: Geophysics, 59, no. 6, 983-999.

Douma, H., \& Baan, M. v. d., 2008, Rational interpolation of qp-traveltimes for semblance-based anisotropy estimation in layered vti media: Geophysics, 73, no. 4, D53-D62.

Douma, H., \& Calvert, A., 2006, Nonhyperbolic moveout analysis in vti media using rational interpolation: Geophysics, 71, no. 3, D59-D71.

Fomel, S., 2004, On anelliptic approximations for qp velocities in vti media: Geophysical Prospecting, 52, no. 3, 247-259.

Gassmann, F., 1964, Introduction to seismic travel time methods in anisotropic media: Pure and Applied Geophysics, 58, no. 1, 63-112.

Gersztenkorn, A., \& Marfurt, K. J., 1999, Eigenstructurebased coherence computations as an aid to 3-d structural and stratigraphic mapping: Geophysics, 64, no. 5, 1468-1479.

Golub, G. H., \& Van Loan, C. F., 1996, Matrix computations. 1996: Johns Hopkins University, Press, Baltimore, MD, USA, pages 374-426.

Grechka, V., \& Tsvankin, I., 1998, Feasibility of nonhyperbolic moveout inversion in transversely isotropic media: Geophysics, 63, no. 3, 957-969.

Jones, L. E., \& Wang, H. F., 1981, Ultrasonic velocities in cretaceous shales from the williston basin: Geophysics, 46, no. 3, 288-297.

Malovichko, A. A., 1978, A new representation of the traveltime curve of reflected waves in horizontally layered media: Applied Geophysics, 91, no. 1, 47-53.

Sarkar, D., Castagna, J. P., \& Lamb, W. J., 2001, Avo and velocity analysis: Geophysics, 66, no. 4, 1284-1293.

Sarkar, D., Baumel, R. T., \& Larner, K. L., 2002, Velocity analysis in the presence of amplitude variation: Geophysics, 67, no. 5, 1664-1672.

Taner, M. T., \& Koehler, F., 1969, Velocity spectra-digital computer derivation applications of velocity functions: Geophysics, 34, no. 6, 859-881.

Thomsen, L., 1986, Weak elastic anisotropy: Geophysics, 51, no. 10, 1954-1966.

Tsvankin, I., \& Thomsen, L., 1994, Nonhyperbolic reflection moveout in anisotropic media: Geophysics, 59, no. 8, 1290-1304.

Ursin, B., Silva, M. G., \& Porsani, M. J., 2014, Signal and traveltime parameter estimation using singular value decomposition: Geophysics, 79, no. 5, U1-U14.

Yan, J., \& Tsvankin, I., 2008, Avo-sensitive semblance analysis for wide-azimuth data: Geophysics, 73, no. 2, U1-U11. 